\title{
Clinical utility gene card for: Maturity-onset diabetes of the young
}

\author{
Kevin Colclough ${ }^{1}$, Cécile Saint-Martin ${ }^{2}$, José Timsit ${ }^{3}$, Sian Ellard ${ }^{1,4}$ and Christine Bellanné-Chantelot ${ }^{\star, 2}$ \\ European Journal of Human Genetics (2014) 22, doi:10.1038/ejhg.2014.14; published online 12 February 2014
}

\section{DISEASE CHARACTERISTICS}

1.1 Name of the disease (synonyms)

Maturity-onset diabetes of the young (MODY); MODY subtype GCK (GCK MODY, MODY2); MODY subtype HNF1A (HNF1A MODY, MODY3); MODY subtype HNF4A (HNF4A MODY, MODY1).

\subsection{OMIM\# of the disease}

606391 (MODY); 125851 (GCK MODY); 600496 (HNF1A MODY); 125850 (HNF4A MODY).

1.3 Name of the analysed genes or DNA/chromosome segments Glucokinase (GCK), hepatocyte nuclear factor 1 alpha (HNF1A) and hepatocyte nuclear factor 4 alpha (HNF4A).

\subsection{OMIM\# of the gene(s)}

138079 (GCK); 142410 (HNF1A); 600281 (HNF4A).

\subsection{Mutational spectrum}

Recent reviews describe 414 different HNF1A mutations in 1247 families, 103 HNF4A mutations in 173 families and 620 GCK mutations in 1441 families. ${ }^{1,2}$

Mutations in these three genes include missense, nonsense, splice site mutations, frameshifts, promoter mutations and in-frame aminoacid deletions, insertions or duplications. These mutations are located throughout the minimal promoter, exons and flanking intronic regions of the GCK, HNF1A and HNF4A genes, although a higher proportion of mutations are found in HNF1A exons 2 and 4, HNF4A exons 7 and 8, and GCK exons 7 and 9. HNF1A missense mutations are more likely to occur within the dimerisation/DNA-binding domains rather than the transactivation domain. Partial and wholegene deletions in GCK, HNF1A and HNF4A are rare and account for $1-3 \%$ of MODY gene mutations. ${ }^{3,4}$

Approximately $60-65 \%$ of mutations identified in GCK, HNF1A and HNF4A are novel, but some mutations are more frequently observed in certain populations because of a founder effect; four ancestral GCK missense mutations (c.118G >A (p.(Glu40Lys)), c.944T >A (p.(Leu315His)), c.952G $>$ A $\quad$ (p. (Gly318Arg)) and c.98T >C (p.(Val33Ala)), nomenclature based on GCK reference sequence NM_000162.3) account for over one-third of GCK MODY families in the Czech population. ${ }^{5}$ A recurrent HNF1A frameshift mutation (c.872dup, p.Gly292fs, nomenclature based on HNF1A reference sequence NM_000545.5) occurs within a polyC tract and accounts for approximately 20\% of published HNF1A MODY families.

The HNF1A and HNF4A genes are highly polymorphic and very rare sequence variants of uncertain clinical significance are frequently identified (a total of 186 polymorphisms/variants of uncertain clinical significance have been published). De novo mutations in $G C K$, HNF1A and HNF4A are rare, but are likely to be underestimated as patients are usually selected for mutation analysis on the basis of their positive family history. ${ }^{6}$

No genotype-phenotype correlation exists for $G C K$ mutations, but the position and the type of HNF1A and HNF4A mutations within the gene influences the age at diagnosis of diabetes in the patient. Patients with missense mutations in the first six exons of the HNF1A gene (predicted to affect all three isoforms) are diagnosed on average 12 years earlier (median of 18 years) than those patients with missense mutations located either in exons 8-10 (affecting isoform A only) or in the transactivation domain (median of 30 years). In contrast, patients with HNF1A truncating mutations are diagnosed at a median age of 20 years independently of the location of the mutation within HNF1A. $^{7,8}$ A similar correlation was observed with HNF4A mutations. ${ }^{9}$

\subsection{Analytical methods}

Mutation screening by direct Sanger sequencing of the coding regions, conserved splice sites and minimal promoter regions is the current gold-standard for mutation detection. Gene dosage analysis using a commercial Multiplex Ligation-dependent Probe Amplification (MLPA) kit is required to identify partial and whole-gene deletions that account for around $1-3 \%$ of mutations. Analysis of the GCK and HNF4A genes should use the pancreatic tissue-specific transcripts (reference sequences NM_000162 for GCK and NM_175914 (LRG_483) for HNF4A). HNF1A analysis should be based on the longest transcript, NM_000545 (LRG_522). Laboratories are encouraged to submit their MODY gene variants to the DMuDB (https:// secure.dmudb.net/ngrl-rep/Home.do) and LOVD (http://grenada. lumc.nl/LOVD2/diabetes/home.php) variant databases.

Genetic testing strategies will vary between laboratories, with analysis of all three genes simultaneously or sequential gene testing based on the clinical features of the patient or the prevalence of each MODY subtype in the population (eg, initial mutation analysis of 
GCK in a patient with mild fasting hyperglycaemia, or only testing for rarer HNF4A mutations if initial screening of HNF1A is negative). Testing for a known mutation can be offered to family members as a diagnostic or predictive test once a molecular genetic diagnosis is obtained for the proband.

Next generation sequencing (NGS) strategies have been employed successfully to identify patients with MODY gene mutations using gene targeted and whole-exome sequencing approaches. ${ }^{10,11}$ NGS allows the simultaneous testing of all known genetic subtypes of MODY (including the rare genetic forms described in section 2.3) that is not possible by Sanger sequencing/MLPA. It is recommended that mutations identified by NGS are confirmed by a second method. Sequence analysis of more genes will result in the identification of more novel (or rare) variants, which require detailed investigation to determine their pathogenicity according to patient phenotype, variant characteristics and co-segregation studies.

\subsection{Analytical validation}

A positive result in a proband can be confirmed by repeat testing of the same DNA sample (routinely undertaken when used as a positive control for family member testing) or from a new DNA extraction (according to laboratory policy). Identification of the mutation in the proband's relatives provides additional confirmation of the result. Barcode tracking and automated liquid/sample handling can reduce the risk of sample transfer errors.

Heterozygous single-nucleotide polymorphisms (SNPs) within PCR primer-binding sites can result in preferential amplification of a single allele and are a known cause of missed mutations. ${ }^{10,12}$ Routine checking of primer-binding sites for SNPs is essential (https://secure.ngrl.org.uk/SNPCheck/snpcheck.htm). This is particularly important in the context of predictive genetic testing; a repeat test with an alternative primer is recommended for patients where the familial mutation is not detected and a SNP within a primer-binding site is identified by the SNP checker tool. Sequence variation in MLPA probe-binding and ligation sites can also cause false-positive results. ${ }^{13}$ Deletions involving a single probe should always be confirmed using a second method.

Quality assurance schemes are available for Sanger sequencing and MODY genetic testing (http://www.emqn.org) and participation is highly recommended to demonstrate that diagnostic testing and reporting procedures meet the required standards.

1.8 Estimated frequency of the disease (incidence at birth ('birth prevalence') or population prevalence. If known to be variable between ethnic groups, please report)

MODY has been well characterised in European and North American populations. Although MODY has been identified in African and Asian populations, the prevalence is not known. Obtaining accurate prevalence data is challenging because of the cost associated with widespread genetic testing of all patients with diabetes and the variable clinical threshold for considering MODY because of overlapping clinical features with more common types of diabetes (type 1 and type 2).

The minimum population prevalence of MODY in the United Kingdom is estimated at 108 cases per million. Studies of populationbased childhood diabetes registries in Norway, Poland and Germany estimated the minimum prevalence of monogenic diabetes as $3.1 / 100000$ in Norwegian children, ${ }^{14} 4.2-4.6 / 100000$ in Polish children, ${ }^{15}$ 2.39/100000 in German children ${ }^{16}$ and 2.1/100000 in American children. ${ }^{17}$
The frequency of the different genetic subtypes is variable and greatly depends on the clinical recruitment (either paediatric or adult) and on the geographic origin of tested patients. In countries such as Italy and the Czech Republic where fasting blood glucose testing is routinely performed in paediatric cases, an incidental finding of mild fasting hyperglycaemia in a child is more likely and results in a higher proportion of GCK MODY being diagnosed. In contrast, HNF1A MODY predominates in countries such as the United Kingdom and Norway, where patients are referred from hospital clinics once a diagnosis of diabetes has been made. Approximately 52\% of confirmed UK MODY families have HNF1A mutations, 32\% have mutations in GCK and $10 \%$ have mutations in HNF4A. ${ }^{18}$ HNF1A mutations are more frequent in Euro-Caucasian patients $(87.5 \%)$ compared with patients with other geographic origins $(22.5 \%) .{ }^{19}$

\subsection{Diagnostic setting}

\begin{tabular}{lll}
\hline & Yes & No \\
A. (Differential) diagnostics & $\bigotimes$ & $\square$ \\
B. Predictive testing & $\Downarrow$ & $\square$ \\
C. Risk assessment in relatives & $\Downarrow$ & $\square$ \\
D. Prenatal & $\square$ & $\square$ \\
\hline
\end{tabular}

Comment: Testing is most commonly undertaken to confirm a diagnosis of MODY and predictive testing for HNF1A and HNF4A MODY is also routinely requested. Predictive testing and the comments in section 3.2 only apply to HNF1A and HNF4A MODY; it is not required for GCK MODY as fasting hyperglycaemia is present from birth and can be ascertained before confirmation with a genetic test. Invasive prenatal testing to predict the future risk of diabetes (or congenital hyperinsulinism for HNF4A mutations) is not indicated. However, if the mother has GCK MODY or either parent has HNF4A MODY, testing of a fetal DNA sample obtained for other purposes (eg, high risk for Down syndrome) is useful for determining the fetal genotype. This predicts fetal size and hence risk of complications during delivery because of macrosomia. ${ }^{20}$ Furthermore, fetal genotyping using cell-free fetal DNA from maternal plasma sampling during early pregnancy is currently being developed to guide management of the pregnancy in mothers with risk of fetal macrosomia because of GCK or HNF4A mutations.

\section{TEST CHARACTERISTICS}

\begin{tabular}{|c|c|c|c|c|}
\hline & \multicolumn{2}{|c|}{ Genotype or disease } & \multirow{2}{*}{$\begin{array}{l}\text { A: True positives } \\
\text { B: False positives }\end{array}$} & \multirow{2}{*}{$\begin{array}{l}\text { C: False negative } \\
\text { D: True negative }\end{array}$} \\
\hline & Present & Absent & & \\
\hline \multicolumn{5}{|l|}{ Test } \\
\hline \multirow[t]{2}{*}{ Positive } & $A$ & $\mathrm{~B}$ & Sensitivity: & $A /(A+C)$ \\
\hline & & & Specificity: & $\mathrm{D} /(\mathrm{D}+\mathrm{B})$ \\
\hline \multirow[t]{2}{*}{ Negative } & C & D & Positive predictive value: & $A /(A+B)$ \\
\hline & & & Negative predictive value: & $D /(C+D)$ \\
\hline
\end{tabular}

\subsection{Analytical sensitivity}

(proportion of positive tests if the genotype is present)

Nearly $100 \%$ for germline mutations in coding regions, flanking intronic regions and minimal promoter regions are analysed by Sanger sequencing, and for gene deletions detected by MLPA if the appropriate gene is analysed. These techniques will not identify deep 
intronic splicing mutations, mutations in as yet unidentified cis-acting regulatory regions and balanced chromosome rearrangements that disrupt the mRNA transcript or separate the promoter from the coding region. ${ }^{21}$ Heterozygous SNPs within PCR primer-binding sites can result in preferential amplification of a single allele and are a known cause of missed mutations.

\subsection{Analytical specificity}

(proportion of negative tests if the genotype is not present)

Nearly $100 \%$. Sequencing artefacts because of non-specific PCR templates can potentially be misinterpreted as mutations. SNPs within MLPA probe-binding sites or probe ligation sites can result in falsepositive dosage results by preventing probe binding and ligation. ${ }^{13}$ Benign, non-pathogenic sequence variants can be reported as MODY causing mutations and careful interpretation taking into account functional studies, segregation analysis, in silico predictions and frequencies according to geographic origin of the patients is required. ${ }^{22}$

\subsection{Clinical sensitivity}

(proportion of positive tests if the disease is present)

The clinical sensitivity can be dependent on variable factors such as age or family history. In such cases, a general statement should be given, even if a quantification can only be made case by case.

The proportion of probands referred for GCK, HNF1A and HNF4A testing in which a confirmed genetic diagnosis of MODY is made varies widely according to the criteria used to select patients for testing. Stricter criteria results in a higher clinical sensitivity, but results in fewer individuals that are diagnosed with MODY.

Clinical sensitivity depends on many factors such as age and phenotype at onset, characteristics of diabetes and associated traits. In patients with a GCK MODY phenotype (ie, mild and stable fasting hyperglycaemia), the pick-up rate of GCK mutations is high ranging from 40 to $50 \%$. In contrast, in patients with a HNF1A or HNF4A MODY phenotype (ie, young-onset, non-insulin-dependent diabetes before 40 years, with a family history of diabetes in at least two generations), HNF1A mutations account for $10-20 \%$ and HNF4A mutations for $10 \%$ of MODY cases without HNF1A mutations.

Although MODY cannot be diagnosed using non-genetic tests alone, a number of inexpensive and widely available biomarkers can help to select patients for genetic testing. GAD and IA2 autoantibodies are present in up to $90-95 \%$ of newly diagnosed type 1 diabetes patients, ${ }^{23}$ but are present in only $1 \%$ of patients with MODY. $^{24}$ Testing for GAD and IA2 auto-antibodies is recommended even long after the onset of diabetes, as recent study showed that at least one antibody (GAD and/or IA2) was present in $56 \%$ of type 1 diabetes patients after a 19-year median duration of the disease. ${ }^{25}$ Urinary and serum C-peptide levels persist in patients with MODY, but are significantly reduced or undetectable in both adult and paediatric type 1 diabetes cases of long duration. ${ }^{26,27}$ Absence of pancreatic auto-antibodies and the detection of C-peptide outside the honeymoon period should therefore raise the suspicion of MODY. Serum high-sensitivity C-reactive protein levels are reduced in HNF1A MODY compared with type 1 and type 2 diabetes, ${ }^{28}$ and can be helpful in discriminating from type 2 diabetes when used in combination with traditional MODY selection criteria. ${ }^{29}$ Features of insulin resistance such as high BMI, hypertension and dyslipidaemia are less prevalent in patients with HNF1A MODY compared with patients with early-onset type 2 diabetes. ${ }^{19}$

Two prediction models have been developed that estimate the prior probability of making a genetic diagnosis of MODY, based on simple clinical parameters. The first model differentiates MODY from type 1 and type 2 diabetes $^{18}$ (online version available at http:// www.diabetesgenes.org/content/mody-probability-calculator) and the second model differentiates HNF1A MODY from early-onset type 2 diabetes. $^{19}$

Mutations in the HNF1B gene (previously named MODY5) are identified in $25 \%$ of patients with young-onset diabetes and renal cysts (RCAD) and/or variable structural abnormalities of the kidney. In about $1 \%$ of cases, $H N F 1 B$ mutations could also be identified in patients with young-onset diabetes who met the criteria of two generations affected with diabetes and at least one family member diagnosed under 25 years and with no known renal disease. ${ }^{30}$

Rarer genetic causes of MODY because of mutations in other genes have been reported, and OMIM lists 11 different subtypes (including GCK, HNF1A and HNF4A MODY). Recent publications of activating KCNJ11 and ABCC8 mutations that cause MODY bring the current total to 13 genes, but these additional subtypes are very rare and together they account for only $\sim 1 \%$ of MODY.

\subsection{Clinical specificity}

\section{(proportion of negative tests if the disease is not present)}

The clinical specificity can be dependent on variable factors such as age or family history. In such cases, a general statement should be given, even if a quantification can only be made case by case.

Nearly, $100 \%$ for GCK MODY. Owing to variability in measuring FBG, $\sim 2 \%$ of patients with GCK MODY have a FBG $<5.5 \mathrm{mmol} / \mathrm{l}$ but on repeat testing it will be $>5.5 \mathrm{mmol} / \mathrm{l}^{31}$ The diabetes in HNF1A and HNF4A MODY is progressive and the penetrance increases with age; for HNF1A mutations, it is approximately $63 \%$ by age 25 years, $93.6 \%$ by age 50 years and $98.7 \%$ by age 75 years. ${ }^{32}$ Patients can therefore have the mutation but be clinically unaffected at a young age, or have biochemical evidence of abnormal glucose homeostasis but be clinically asymptomatic. Patients with HNF1A and HNF4A mutations can have FBG within the non-diabetic range, but have oral glucose tolerance test results diagnostic for diabetes. $^{31}$

Diabetic family members of a proband with MODY may not inherit the familial mutation, and their diabetes is likely to have a different aetiology (typically type 1 or type 2 diabetes).

\subsection{Positive clinical predictive value}

(life-time risk to develop the disease if the test is positive)

GCK MODY is characterised by fasting hyperglycaemia, which is present from birth. The penetrance of HNF1A and HNF4A MODY is age related, and increases with age (see section 2.4).

\subsection{Negative clinical predictive value}

(probability not to develop the disease if the test is negative)

Assume an increased risk based on family history for a non-affected person. Allelic and locus heterogeneity may need to be considered.

Index case in that family had been tested:

Nearly $100 \%$ probability not to develop MODY, although the individual is still at population risk of developing type 1 or type 2 diabetes. Testing will not exclude the possibility of developing MODY because of other MODY gene mutations that were not tested.

Index case in that family had not been tested:

Testing for a clinically unaffected individual would only be undertaken if the familial MODY causing mutation has been identified. 


\section{CLINICAL UTILITY}

3.1 (Differential) diagnostics: The tested person is clinically affected (To be answered if in 1.9 'A' was marked)

\subsubsection{Can a diagnosis be made other than through a genetic test?}

\begin{tabular}{|c|c|c|}
\hline No & $\otimes$ (continue with 3.1 .4 ) & \\
\hline \multirow[t]{7}{*}{ Yes } & $\square$ & \\
\hline & Clinically & $\square$ \\
\hline & Imaging & $\square$ \\
\hline & Endoscopy & $\square$ \\
\hline & Biochemistry & $\square$ \\
\hline & Electrophysiology & $\square$ \\
\hline & Other (please describe) & \\
\hline
\end{tabular}

\subsubsection{Describe the burden of alternative diagnostic methods to the} patient

Not applicable.

\subsubsection{How is the cost effectiveness of alternative diagnostic methods to be judged?}

Not applicable.

\subsubsection{Will disease management be influenced by the result of a} genetic test?

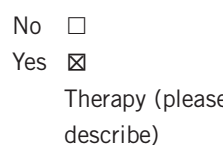

describe)

Prognosis (please describe)
Low-dose sulfonylureas are recommended as the firstline treatment for patients with HNF1A/HNF4A-MODY and observational evidence suggests that patients can be switched safely from insulin to a sulfonylurea. ${ }^{33}$ In a series of 43 diabetic patients, 34 changed from insulin to a sulfonylurea after diagnosis of HNF1A MODY and 24 remained off insulin for 39 months with no deterioration in glycaemic control. ${ }^{34}$ Successful transfer was correlated with a shorter duration of diabetes and emphasises the need for early genetic diagnosis. Good control may be maintained for many years, although eventually most patients progress to insulin treatment.

Hypersensitivity to sulphonylureas can result in symptomatic hypoglycaemia for some HNF1A and HNF4A MODY patients. Treating these patients with a short acting insulin secretagogue such as nateglinide has been shown to be a safe alternative therapy that results in less hypoglycaemic symptoms. ${ }^{35}$ The majority of patients with GCK MODY are not receiving pharmacological therapy but some are misdiagnosed with type 1 or 2 diabetes and treated with insulin or oral hypoglycaemia agents. No change in $\mathrm{HbA} 1 \mathrm{c}$ was seen in a longitudinal study of 16 subjects who stopped treatment after their GCK mutation was identified. ${ }^{36}$ Therefore, in the absence of concomitant type 1 or 2 diabetes, glucose-lowering therapy can be stopped. The only exception is during a pregnancy where fetal macrosomia is suspected and insulin treatment may be administered in an attempt to control fetal growth.

Due to its progressive nature and early age at diagnosis, those with HNF1A and HNF4A MODY are at similar risk of microvascular and macrovascular complications, as patients with type 1 and type 2 diabetes, which is strongly influenced by the degree of glycaemic control. HNF1A MODY patients are at an increased risk of developing cardiovascular disease so statin therapy by the age of 40 years, regardless of lipid status, has been recommended. ${ }^{37}$ The fasting hyperglycaemia in GCK MODY is mild and stable over the patient's lifetime, with progression reflecting that seen with increasing age in the non-GCK MODY population. As $\mathrm{HbAlc}$ is only mildly raised, the risk of developing diabetic complications is very low. 38

Management (please Women with GCK MODY may have babies that are large describe) for gestational age if the baby has not inherited the GCK mutation. It is suggested that mothers undergo serial fetal abdominal scans to identify babies showing excessive growth to thereby target insulin therapy to those at greatest risk. Finding an HNF4A mutation has important implications for the management of pregnancy. Pregnancies where one parent (mother or father) has an HNF4A mutation are at risk of complications during delivery because of macrosomia and increased ultrasound monitoring of fetal growth is recommended. ${ }^{39}$ Neonates who have inherited the HNF4A mutation are also at risk of neonatal hyperinsulinaemic hypoglycaemia and blood glucose should be checked from birth. ${ }^{39}$

HNF1A mutations may predispose to familial liver adenomatosis through somatic inactivation of the remaining wild-type allele. In a cohort of $137 \mathrm{HNF} 1 \mathrm{~A}$ MODY patients, 9 cases (6.5\%) of liver adenomatosis were identified by liver ultrasonography (Rod et al, personal communication). Patients with liver adenomatosis are at risk of haemorrhage, and some hepatologists advocate systematic liver ultrasonography in HNF1A MODY patients.

3.2 Predictive setting: The tested person is clinically unaffected but carries an increased risk based on family history (To be answered if in 1.9 'B' was marked)

\subsubsection{Will the result of a genetic test influence lifestyle and} prevention?

If the test result is positive (please describe):

Annual screening for diabetes in asymptomatic mutation carriers is recommended from the age of 10 years. Maintaining normal body weight is also recommended as a high BMI is associated with an earlier age of onset of diabetes. ${ }^{40}$

If the test result is negative (please describe):

If the familial mutation is not identified, the individual is not genetically predisposed to MODY and their risk of developing diabetes is reduced to that of the population. Screening for diabetes should reflect general practice guidelines in light of other clinical risk factors.

3.2.2 Which options in view of lifestyle and prevention does a person at-risk have if no genetic test has been done? At-risk individuals can be monitored for the development of diabetes whether they have the genetic test or not.

3.3 Genetic risk assessment in family members of a diseased person (To be answered if in 1.9 'C' was marked) 
3.3.1 Does the result of a genetic test resolve the genetic situation in that family?

In most cases yes, although the presence of other rare MODY gene mutations/mutations in genes not yet identified cannot be excluded. The risk of identifying a novel or rare variant of uncertain significance is similar to most other genetic disorders and may take time to resolve/leave uncertainty around the diagnosis.

\subsubsection{Can a genetic test in the index patient save genetic or other} tests in family members?

If a MODY gene mutation is identified in the index patient, confirmatory genetic testing should be offered to first-degree diabetic relatives in the first instance. It is not appropriate to assume that other diabetic relatives of the index patient are also affected with MODY, as type 1 diabetes and type 2 diabetes can co-exist in MODY families and HNF1A-MODY is often misdiagnosed as type 1 diabetes or type 2 diabetes. ${ }^{19}$ Genetic confirmation of MODY and assessment of endogenous insulin production in insulin-treated patients is essential before undertaking a change in treatment.

If no mutation is identified in the index patient, a diagnosis of MODY in family is unlikely, but cannot be excluded because of rare mutations in other MODY genes, or genes that have yet to be identified. Testing should always be undertaken on the family member clinically most likely to have MODY.

3.3.3 Does a positive genetic test result in the index patient enable a predictive test in a family member?

First-degree relatives of probands with HNF1A and HNF4A MODY who are not known to have diabetes but have requested genetic testing may be tested initially by performing fasting blood glucose, HbA1C and/or an oral glucose tolerance test. Referral to Clinical Genetics is recommended for family members without recognised diabetes who request predictive molecular genetic testing.

Unaffected family members of probands with GCK MODY may be tested initially by measuring their fasting blood glucose; if elevated ( $>5.5 \mathrm{mmol} / \mathrm{l}$ ), molecular genetic testing can then be performed to confirm a diagnosis of GCK MODY.

\subsection{Prenatal diagnosis}

(To be answered if in 1.9 "D" was marked)

\subsubsection{Does a positive genetic test result in the index patient enable a prenatal diagnosis?}

Not applicable.

\section{IF APPLICABLE, FURTHER CONSEQUENCES OF TESTING}

Please assume that the result of a genetic test has no immediate medical consequences. Is there any evidence that a genetic test is nevertheless useful for the patient or his/her relatives?

A positive genetic test confirms the aetiology of the patient's diabetes and informs the prognosis of the disease. It also alerts clinicians as to the risk of developing other non-diabetic clinical features, such as liver adenomas and cardiovascular disease in HNF1A MODY. Finding a GCK or an HNF4A mutation has implications for the management and outcomes of pregnancy.

It explains the history of diabetes in the family, and allows for the correct diagnosis in other affected relatives who may be misclassified and not adequately treated. It enables predictive testing of asymptomatic individuals to remove the uncertainty of developing diabetes and to identify subjects with subclinical diabetes. Individuals with a MODY gene mutation can be informed of the risk of their offspring inheriting the mutation and developing diabetes or having fasting hyperglycaemia.

\section{CONFLICT OF INTEREST}

The authors declare no conflict of interest.

\section{ACKNOWLEDGEMENTS}

This work was supported by EuroGentest2 (Unit 2: 'Genetic testing as part of health care'), a Coordination Action under FP7 (Grant Agreement Number 261469) and the European Society of Human Genetics.

1 Colclough K, Bellanne-Chantelot C, Saint-Martin C, Flanagan SE, Ellard S: Mutations in the genes encoding the transcription factors hepatocyte nuclear factor 1 alpha and 4 alpha in maturity-onset diabetes of the young and hyperinsulinemic hypoglycemia. Hum Mutat 2013; 34: 669-685.

2 Osbak KK, Colclough K, Saint-Martin C et al: Update on mutations in glucokinase (GCK), which cause maturity-onset diabetes of the young, permanent neonatal diabetes, and hyperinsulinemic hypoglycemia. Hum Mutat 2009; 30: 1512-1526.

3 Carette C, Dubois-Laforgue D, Saint-Martin C et al: Familial young-onset forms of diabetes related to HNF4A and rare HNF1A molecular aetiologies. Diabet Med 2010; 27: $1454-1458$

4 Ellard S, Thomas K, Edghill EL et al: Partial and whole gene deletion mutations of the GCK and HNF1A genes in maturity-onset diabetes of the young. Diabetologia 2007; 50: 2313-2317.

5 Dusatkova P, Pruhova S, Borowiec M et al: Ancestral mutations may cause a significant proportion of GCK-MODY. Pediatr Diabetes 2012; 13: 489-498.

6 Stanik J, Dusatkova P, Cinek 0 et al: De novo mutations of GCK, HNF1A and HNF4A may be more frequent in MODY than previously assumed. Diabetologia 2013; e-pub ahead of print 10 December 2013.

7 Bellanne-Chantelot C, Carette C, Riveline JP et al: The type and the position of HNF1A mutation modulate age at diagnosis of diabetes in patients with maturity-onset diabetes of the young (MODY)-3. Diabetes 2008; 57: 503-508.

8 Harries LW, Ellard S, Stride A, Morgan NG, Hattersley AT: Isomers of the TCF1 gene encoding hepatocyte nuclear factor-1 alpha show differential expression in the pancreas and define the relationship between mutation position and clinical phenotype in monogenic diabetes. Hum Mol Genet 2006; 15: 2216-2224.

9 Harries LW, Locke JM, Shields B et al: The diabetic phenotype in HNF4A mutation carriers is moderated by the expression of HNF4A isoforms from the P1 promoter during fetal development. Diabetes 2008; 57: 1745-1752.

10 Ellard S, Lango Allen H, De Franco $\mathrm{E}$ et al: Improved genetic testing for monogenic diabetes using targeted next-generation sequencing. Diabetologia 2013; 56 : 1958-1963

11 Johansson S, Irgens $\mathrm{H}$, Chudasama KK et al: Exome sequencing and genetic testing for MODY. PLoS One 2012; 7: e38050.

12 Raeder H, Bjorkhaug L, Johansson S et al: A hepatocyte nuclear factor-4 alpha gene (HNF4A) P2 promoter haplotype linked with late-onset diabetes: studies of HNF4A variants in the Norwegian MODY registry. Diabetes 2006; 55: 1899-1903.

13 Thanabalasingham G, Kaur K, Talbot F et al: Atypical phenotype associated with reported GCK exon 10 deletions: Clinical judgement is needed alongside appropriate genetic investigations. Diabet Med 2013; 30: e233-e238.

14 Irgens HU, Molnes J, Johansson BB et al: Prevalence of monogenic diabetes in the population-based Norwegian Childhood Diabetes Registry. Diabetologia 2013; 56 : 1512-1519.

15 Fendler W, Borowiec M, Baranowska-Jazwiecka A et al: Prevalence of monogenic diabetes amongst Polish children after a nationwide genetic screening campaign. Diabetologia 2012; 55: 2631-2635.

16 Neu A, Feldhahn L, Ehehalt S, Hub R, Ranke MB: Type 2 diabetes mellitus in children and adolescents is still a rare disease in Germany: a population-based assessment of the prevalence of type 2 diabetes and MODY in patients aged 0-20 years. Pediatr Diabetes 2009; 10: 468-473.

17 Gilliam LK, Pihoker C, Ellard S et al: Prevalence, Characteristics and Clinical Diagnosis of Maturity Onset Diabetes of the Young Due to Mutations in HNF1A, HNF4A, and Glucokinase: Results from the SEARCH for Diabetes in Youth. J Clin Endocrinol Metab 2013; 98: 4055-4062.

18 Shields BM, McDonald TJ, Ellard S, Campbell MJ, Hyde C, Hattersley AT: The development and validation of a clinical prediction model to determine the probability of MODY in patients with young-onset diabetes. Diabetologia 2012; 55: 1265-1272.

19 Bellanne-Chantelot C, Levy DJ, Carette C et al: Clinical Characteristics and Diagnostic Criteria of Maturity-Onset Diabetes Of The Young (MODY) due to Molecular Anomalies of the HNF1A Gene. J Clin Endocrinol Metab 2011; 96: E1346-E1351.

20 Chakera AJ, Carleton VL, Ellard S et al: Antenatal diagnosis of fetal genotype determines if maternal hyperglycemia due to a glucokinase mutation requires treatment. Diabetes Care 2012; 35: 1832-1834.

21 Gloyn AL, Ellard S, Shepherd M et al: Maturity-onset diabetes of the young caused by a balanced translocation where the $20 \mathrm{q} 12$ break point results in disruption upstream of the coding region of hepatocyte nuclear factor-4alpha (HNF4A) gene. Diabetes 2002; 51: 2329-2333 
22 Steele AM, Tribble ND, Caswell $\mathrm{R}$ et al: The previously reported T342P GCK missense variant is not a pathogenic mutation causing MODY. Diabetologia 2011; 54 2202-2205.

23 Gorus FK, Goubert P, Semakula C et al: IA-2-autoantibodies complement GAD65-autoantibodies in new-onset IDDM patients and help predict impending diabetes in their siblings. The Belgian Diabetes Registry. Diabetologia 1997; 40: 95-99.

24 McDonald TJ, Colclough K, Brown R et al: Islet autoantibodies can discriminate maturity-onset diabetes of the young (MODY) from Type 1 diabetes. Diabet Med 2011; 28: 1028-1033.

25 Wilmot-Roussel H, Levy DJ, Carette $\mathrm{C}$ et al: Factors associated with the presence of glutamic acid decarboxylase and islet antigen-2 autoantibodies in patients with longstanding type 1 diabetes. Diabetes Metab 2013; 39: 244-249.

26 Besser RE, Shepherd MH, McDonald TJ et al: Urinary C-peptide creatinine ratio is a practical outpatient tool for identifying hepatocyte nuclear factor 1 -\{alpha\}/hepatocyte nuclear factor 4-\{alpha\} maturity-onset diabetes of the young from long-duration type 1 diabetes. Diabetes Care 2011; 34: 286-291.

27 Besser RE, Shields BM, Hammersley SE et al: Home urine C-peptide creatinine ratio (UCPCR) testing can identify type 2 and MODY in pediatric diabetes. Pediatr Diabetes 2013; 14: 181-188.

28 McDonald TJ, Shields BM, Lawry J et al: High-sensitivity CRP discriminates HNF1A-MODY from other subtypes of diabetes. Diabetes Care 2011; 34: 1860-1862.

29 Owen KR, Thanabalasingham G, James TJ et al: Assessment of high-sensitivity C-reactive protein levels as diagnostic discriminator of maturity-onset diabetes of the young due to HNF1A mutations. Diabetes Care 2010; 33: 1919-1924.

30 Edghill EL, Stals K, Oram RA, Shepherd MH, Hattersley AT, Ellard S: HNF1B deletions in patients with young-onset diabetes but no known renal disease. Diabet Med 2013; 30: 114-117.
31 Stride A, Vaxillaire M, Tuomi $\mathrm{T}$ et al: The genetic abnormality in the beta cell determines the response to an oral glucose load. Diabetologia 2002; 45: 427-435.

32 Shepherd M, Ellis I, Ahmad AM et al: Predictive genetic testing in maturity-onset diabetes of the young (MODY). Diabet Med 2001; 18: 417-421.

33 Pearson ER, Starkey BJ, Powell RJ, Gribble FM, Clark PM, Hattersley AT: Genetic cause of hyperglycaemia and response to treatment in diabetes. Lancet 2003; 362 . 1275-1281.

34 Shepherd M, Shields B, Ellard S, Rubio-Cabezas O, Hattersley AT: A genetic diagnosis of HNF1A diabetes alters treatment and improves glycaemic control in the majority of insulin-treated patients. Diabet Med 2009; 26: 437-441.

35 Tuomi T, Honkanen EH, Isomaa B, Sarelin L, Groop LC: Improved prandial glucose control with lower risk of hypoglycemia with nateglinide than with glibenclamide in patients with maturity-onset diabetes of the young type 3. Diabetes Care 2006; 29: 189-194.

36 Stride A, Shields B, Gill-Carey 0 et al: Cross-sectional and longitudinal studies suggest pharmacological treatment used in patients with glucokinase mutations does not alter glycaemia. Diabetologia 2014; 57: 54-56

37 Steele AM, Shields BM, Shepherd M, Ellard S, Hattersley AT, Pearson ER: Increased all-cause and cardiovascular mortality in monogenic diabetes as a result of mutations in the HNF1A gene. Diabet Med 2010; 27: 157-161.

38 Steele AM, Wensley KJ, Ellard S et al: Use of HbAlc in the identification of patients with hyperglycaemia caused by a glucokinase mutation: observational case control studies. PLoS One 2013; 8: e65326.

39 Pearson ER, Boj SF, Steele AM et al: Macrosomia and hyperinsulinaemic hypoglycaemia in patients with heterozygous mutations in the HNF4A gene. PLoS Med 2007; 4: e118.

40 Lehto M, Tuomi T, Mahtani MM et al: Characterization of the MODY3 phenotype. Early-onset diabetes caused by an insulin secretion defect. J Clin Invest 1997; 99 582-591. 